\title{
Diffusion and Segmental Dynamics of Double-Stranded DNA
}

\author{
E. P. Petrov, ${ }^{1}$ T. Ohrt, ${ }^{1}$ R. G. Winkler, ${ }^{2}$ and P. Schwille ${ }^{1}$ \\ ${ }^{1}$ Institute of Biophysics/BIOTEC, Dresden University of Technology, Tatzberg 47-51, 01307 Dresden, Germany \\ ${ }^{2}$ Institute of Solid State Research, Research Center Jülich, 52425 Jülich, Germany
}

(Received 28 January 2006; published 18 December 2006)

\begin{abstract}
Diffusion and segmental dynamics of the double-stranded $\lambda$-phage DNA polymer are quantitatively studied over the transition range from stiff to semiflexible chains. Spectroscopy of fluorescence fluctuations of single-end fluorescently labeled monodisperse DNA fragments unambiguously shows that doublestranded DNA in the length range of $10^{2}-2 \times 10^{4}$ base pairs behaves as a semiflexible polymer with segmental dynamics controlled by hydrodynamic interactions.
\end{abstract}

The dynamic behavior of individual macromolecules in solution is governed by chain connectivity and hydrodynamic interactions [1]. Understanding polymer dynamics and quantitative verification of polymer theories requires detailed information on segmental motion of individual polymer molecules. However, the classical experimental techniques, such as dynamic light scattering (DLS) or transient electric birefringence (TEB), predominantly deliver information on large-scale shape fluctuations of macromolecules, and development of new experimental approaches providing detailed information on internal dynamics of individual molecules is of high importance.

Precise experiments in polymer physics are impossible without well-defined monodisperse polymer samples covering a wide range of molecular weights. The recent progress in molecular biotechnology resulted in a variety of techniques to produce monodisperse DNA fragments, which stimulated the use of DNA as a model compound in studies of polymer dynamics in solution [2]. Doublestranded DNA (dsDNA) is a biopolymer characterized by a large persistence length $l_{p} \sim 50 \mathrm{~nm}$ [3]. As a result, dsDNA fragments exhibit rodlike, semiflexible, or even flexible polymer behavior, depending on their length. Thus, simple generic models of polymer dynamics [1] are not expected to provide a quantitative description of dsDNA behavior, and more advanced models accounting for the persistence of the polymer chain [4] are required.

The dynamics of dsDNA in solution has been a subject of a number of experimental investigations carried out by various techniques, including DLS [5], single-molecule fluorescence microscopy [6], electrophoresis [7], and TEB [8]. Fluorescence correlation spectroscopy (FCS) [9], a single-molecule technique that can provide more detailed information on the macromolecular dynamics than the classical ensemble-based methods, has been recently applied to investigation of DNA in solution [10-12], which has lead to a controversy whether dsDNA dynamics in dilute solution is controlled by hydrodynamic interactions [10,12] or not [11]. Apart from this controversy, for different reasons, none of these FCS-based experiments produced parameters of DNA dynamics. Generally, to the best of our knowledge, no experimental studies were published so far where diffusion and intramolecular dynamics of dsDNA have been simultaneously investigated over the transition range from stiff to semiflexible chains. In the present Letter, we fill this gap and carry out a study of the solution dynamics of single-end fluorescently labeled dsDNA fragments in the range of lengths $L$ from $10^{2}$ to $2 \times 10^{4}$ base pairs (bp) $\left(L / l_{p} \sim 0.7-140\right)$ by following the Brownian motion of the labeled ends of single DNA molecules using fluorescence correlation spectroscopy. Diffusion coefficients of dsDNA fragments obtained from FCS data in a model-independent way, as well as polymer relaxation times obtained by applying the theory of semiflexible polymer dynamics [4] to experimental data, show excellent agreement with both independent experimental data and theory. Our results clearly demonstrate that in this length range dsDNA behaves as a semiflexible polymer with strong hydrodynamic interactions.

We employ the technique of the polymerase chain reaction (PCR) [13] to produce monodisperse samples of DNA fragments with the predefined sequence, structure, and length determined by the forward and reverse primers [14] using $\lambda$-phage DNA (Fermentas Life Sciences, SD0021) as a template. DNA samples fluorescently labeled at the same single end were produced by using the forward primer labeled at its $5^{\prime}$ end with Alexa 488 dye (Molecular Probes) [15] via a $\mathrm{C}_{6}$-amino linker. Reverse primers were selected to produce DNA fragments with the lengths of 0.1 , $0.2,0.5,1,2,5,10$, and $20 \mathrm{kbp}$. All primers were obtained from IBA GmbH. In contrast to the approach used in [11], our PCR-based procedure [16] provides true single labeling and excludes the presence of unlabeled or double-endlabeled DNA fragments.

FCS [9] is a powerful experimental technique to study diffusional motion, intramolecular dynamics, and chemical reactions of fluorescently labeled molecules at nanomolar concentrations under (quasi)equilibrium conditions in solutions and cellular systems. The method consists in studying fluctuations of the confocally detected fluorescence signal $F(t)=\langle F\rangle+\delta F(t)$ via the correlation function $G(\tau)=\langle\delta F(t) \delta F(t+\tau)\rangle /\langle F\rangle^{2}$. In the absence of addi- 
tional photophysical processes and chemical reactions, the fluorescence signal fluctuates as a result of the Brownian motion of fluorescently labeled particles through a femtoliter-sized confocal detection volume whose shape can be well approximated by a 3D Gaussian $\exp \left(-2 r^{2} / r_{0}^{2}-2 z^{2} / z_{0}^{2}\right)$ [17] with the lateral and axial extensions $r_{0}$ and $z_{0}$. If the Brownian motion of a fluorophore is characterized by a Gaussian distribution of displacements with the mean-square displacement (MSD) $\left\langle\mathbf{r}^{2}(t)\right\rangle$, the FCS correlation function is

$$
G(t)=\frac{1}{\langle N\rangle} \frac{1}{\left(1+\frac{2}{3} \frac{\left\langle\mathbf{r}^{2}(t)\right\rangle}{r_{0}^{2}}\right) \sqrt{1+\frac{2}{3} \frac{\left.\left\langle\mathbf{r}^{2}(t)\right)\right\rangle}{z_{0}^{2}}}},
$$

where $\langle N\rangle$ is the effective number of fluorescent molecules in the detection volume.

FCS experiments were carried out on a ConfoCor2 setup (Zeiss) [18] (excitation: Ar-ion laser, $488 \mathrm{~nm}$; objective: CApochromat $40 \times \mathrm{NA} 1.2 \mathrm{~W}$ (Zeiss); pinhole: $90 \mu \mathrm{m}$; fluorescence detection range: $505-550 \mathrm{~nm}$ ). FCS data were recorded as sequences of photon arrival times and processed using a software correlator. The laser intensity at the sample was $1.2 \mu \mathrm{W}$. At this intensity, the effects of triplet blinking [19] and photobleaching [20] on the shape of FCS curves were found to be negligible. The detector afterpulsing was removed according to Ref. [21]. To avoid artifacts due to evaporation-induced convection, samples were hermetically sealed between two cover glasses using a silicone spacer. Concentrations of DNA estimated from the background-corrected amplitudes of the FCS curves [9] were in agreement with results of spectrophotometric measurements [22], and were well below the corresponding coil overlap concentrations [1]. All measurements were carried out at $25.0 \pm 0.5^{\circ} \mathrm{C}$.

The FCS setup was calibrated using an aqueous solution of freely diffusing rhodamine 6G dye (Lambda Physik) [diffusion coefficient $(4.14 \pm 0.1) \times 10^{-10} \mathrm{~m}^{2} \mathrm{~s}^{-1}$ in water at $25^{\circ} \mathrm{C}$ [23] ] or Alexa 488 (the same value under identical conditions). This allowed us to determine the parameters of the fluorescence detection volume: $r_{0} \approx$ $0.20 \mu \mathrm{m}$ and $z_{0} / r_{0}=5.6 \pm 0.1$.

To facilitate a model-independent analysis of experimental data, FCS correlation functions were normalized by the respective amplitudes [24], and MSDs of fluorescently labeled DNA ends were estimated by inverting Eq. (1). Equation (1) is strictly valid only if the distribution of displacements of the fluorescently labeled polymer end group is Gaussian. The validity of this assumption has been confirmed for a wide range of polymer lengths and flexibilities [25]. We find (Fig. 1) that for the relatively small molecules the correlation curves and the reconstructed MSDs follow the expected behavior for free diffusion in three dimensions $(\mathrm{MSD} \sim t)$. For DNA fragments with lengths $\geq 0.2 \mathrm{kbp}$, the short-time dynamics of the labeled end deviates from free diffusion and approximately follows a power law $\sim t^{\beta}$. At longer times, as expected, the end

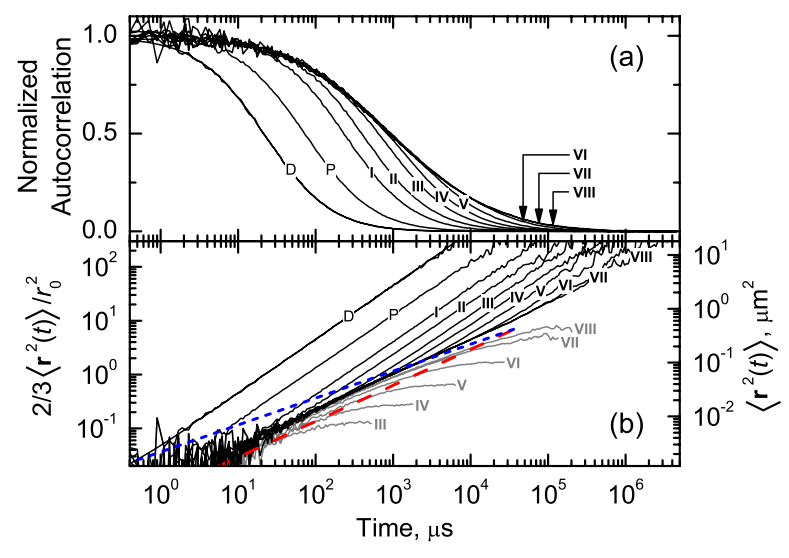

FIG. 1 (color online). Normalized FCS correlation functions (a) and calculated reduced mean-square displacements (b) of freely diffusing Alexa 488 dye (D), labeled forward primer (P), and single-end-labeled $\lambda$-DNA fragments with lengths of 0.1 (I), 0.2 (II), 0.5 (III), 1 (IV), 2 (V), 5 (VI), 10 (VII), and $20 \mathrm{kbp}$ (VIII). Also shown are the fit of Alexa 488 data to the free diffusion model (a) and the corresponding MSD (b). Panel (b) additionally shows intramolecular contributions to the MSD (gray curves) obtained by subtracting the contributions due to overall Fickian diffusion of dsDNA polymers from the corresponding data, as well as $t^{2 / 3}$ (long dashed line) and $t^{1 / 2}$ (short dashed line) power laws. The erratic deviations of the curves reflect statistical errors in the data.

segments exhibit Fickian diffusion due to the translational Brownian motion of the whole macromolecule.

For long flexible polymers, the Rouse and Zimm models predict the short-time behavior of the MSD of the polymer end to follow a power law $\sim t^{\beta}$ with exponents $\beta=1 / 2$ (free-draining regime) and $2 / 3$ (nondraining regime), respectively [1]; for semiflexible polymers, the short-time behavior with $\beta=3 / 4$ is expected $[4,26]$. One should keep in mind, however, that these power laws are valid only for times much shorter than the longest relaxation time of the polymer, whereas at comparable and longer times the intramolecular contribution to the MSD saturates due to the finite size of the polymer coil [Fig. 1(b), gray curves]. Thus, direct application of the above power laws to the analysis of the MSD time dependences, as has been recently proposed in Ref. [11], can be misleading. Indeed, even for the longest sample the apparent power law in $\left\langle\mathbf{r}^{2}(t)\right\rangle$ observed in the range of $10^{0}-10^{5} \mu$ s is to a large extent due to the crossover from intramolecular motion to overall polymer diffusion [Fig. 1(b)]; only for times shorter than $\sim 10^{3} \mu$ s can one observe a power-law-type behavior in the intramolecular contribution. For shorter DNA samples, the time range of the power-law-type segmental dynamics shifts to even shorter times. As a result, the direct analysis of the MSD of a labeled polymer segment should be carried out with extreme caution and applied only to time ranges much shorter than the longest polymer relaxation time. We would like to point out that such an analysis of the short-time dynamics, still possible in our study for the two longest DNA fragments, clearly rules out the 
Rouse-type behavior reported for dsDNA in [11] and leads to a definitive conclusion on the importance of intramolecular hydrodynamic interactions in dsDNA polymer dynamics.

To extract quantitative results on polymer dynamics, a model describing the experimental FCS correlation functions is required. We carry out such a quantitative analysis on the basis of semiflexible polymer theory [4], where we use Eq. (1) with the MSD of the labeled DNA end given by a sum of contributions due to translational diffusion and intramolecular dynamics $[4,12]$ :

$$
\left\langle\mathbf{r}^{2}(t)\right\rangle=6 D t+\frac{2 k_{B} T}{\pi \eta} \sum_{l=1}^{\infty} \tau_{l} \psi_{l}^{2}(L / 2)\left(1-e^{-t / \tilde{\tau}_{l}}\right),
$$

where $T$ is the temperature, $k_{B}$ the Boltzmann factor, and $\eta$ the viscosity. $\psi_{l}(L / 2)$ is the value of the $l$ th eigenfunction of a chain of length $L$ at its end, $\tilde{\tau}_{l}$ is the $l$ th relaxation time in the presence of hydrodynamic interactions, $\tau_{l}$ is the freedraining relaxation time, and $D$ is the translational diffusion coefficient of the macromolecule. For details, see [4]. The two relaxation times are related via $\tilde{\tau}_{l}=\tau_{l} /(1+$ $\left.3 \pi \eta \Lambda H_{l l}\right)$ and $D=k_{B} T\left(1+\Lambda_{D} H_{00}\right) / 3 \pi \eta L$, where $H_{l l}$ $(l \geq 0)$ are the matrix elements of the Rotne-Prager tensor $\mathbf{H}$ in terms of the eigenfunctions $\psi_{l}$ within the preaveraging approximation [4]. The correction factors $\Lambda$ and $\Lambda_{D}$ are introduced in our quantitative analysis of the experimental data to account for the effect of the approximations employed, which lead to an overestimation of the diffusion coefficient and underestimation of the relaxation times (p. 106 of Ref. [1], and references therein). Nonlinear weighted least-squares fitting of normalized FCS curves was carried out by setting $\langle N\rangle=1.0$ and using the persistence length $l_{p}$ and the coefficients $\Lambda$ and $\Lambda_{D}$ as free parameters [27]. In the calculations we used the interbase-pair distance of $0.34 \mathrm{~nm} / \mathrm{bp}$ and DNA thickness of $2.5 \mathrm{~nm}$ [3]. As exemplified in Fig. 2, for dsDNA fragments with lengths within the range of $0.5-20 \mathrm{kbp}$ the model reproduces the shape of the FCS curves extremely well, providing the mean persistence length value of $51 \pm 1 \mathrm{~nm}$, in agreement with literature data. The correction coefficients are $\Lambda \approx 0.6$ and $\Lambda_{D} \approx 0.9$, which is equivalent to a $40 \%$ and $10 \%$ reduction in the hydrodynamic interactions affecting $\tilde{\tau}_{l}$ and $D$, respectively. Considering the simplicity

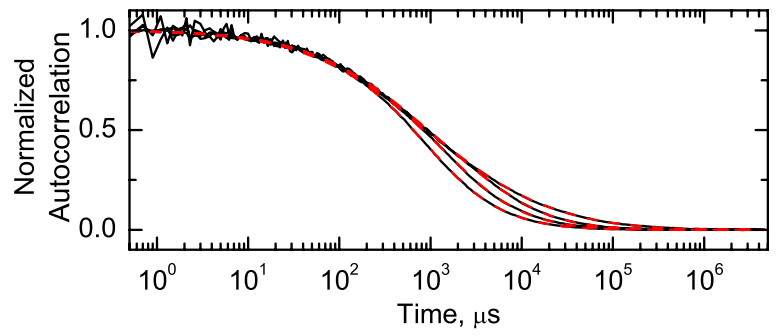

FIG. 2 (color online). Normalized FCS autocorrelation functions of $0.5,1,2$, and $20 \mathrm{kbp}$ DNA fragments (left to right) and their fits by Eqs. (1) and (2) (dashed line). of the model and the approximations involved (preaveraging, neglecting off-diagonal elements of $\mathbf{H}$, no excludedvolume interactions), these are small quantitative deviations from the theoretical predictions. The difference between $\Lambda$ and $\Lambda_{D}$ could indicate a somewhat stronger influence of the approximations on $\tilde{\tau}_{l}$ than on $D$. Notice that the effect of $\Lambda$ and $\Lambda_{D}$ on the dynamic parameters becomes less pronounced with decreasing fragment length.

Figure 3 displays the obtained diffusion coefficients $D$ [28] and the longest relaxation times $\tau_{r}=\tilde{\tau}_{1}$ for dsDNA fragments in a wide range of lengths; the upper part of this range is not readily accessible to the classical techniques like DLS or TEB. Within the range of lengths studied, $D \sim$ $L^{-2 / 3}$ and $\tau_{r} \sim L^{2}$, and thus the results do not follow the predictions of either the Rouse or Zimm model [1]. At the same time, our data are in a very good agreement with experimental results obtained by other techniques not related to FCS [5-8] and closely follow the predictions of the semiflexible polymer theory [4].

Therefore, our results clearly demonstrate that in the range of the lengths studied, dsDNA behaves as a semiflexible polymer with strong hydrodynamic interactions [29]. The Zimm regime with MSD $\sim t^{2 / 3}, D \sim L^{-1 / 2}$, and $\tau_{r} \sim L^{3 / 2}$ (or corresponding expressions modified to account for excluded-volume interactions) [1] can be achieved only for long dsDNA molecules with lengths exceeding $10^{5} \mathrm{bp}$, or $\sim 10^{3} l_{p}$ (Fig. 3).

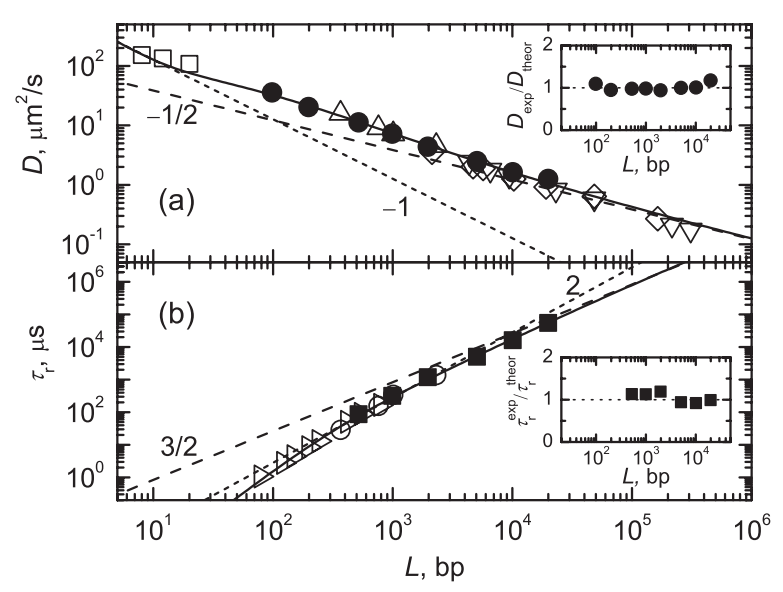

FIG. 3. Diffusion coefficients (a) and longest relaxation times (b) of dsDNA. Present work (corrected to $\left.20^{\circ} \mathrm{C}\right)($ $\square)$, data from Refs. [5(a)] ( $\square)$, [5(b)] ( $\triangle)$, [6] $(\nabla)$, [7] $(\diamond)$, $[8(\mathrm{a})](\bigcirc),[8(\mathrm{~b})](\triangleright)$. Predictions of the semiflexible polymer theory [4] for $l_{p}=50 \mathrm{~nm}, \Lambda_{D}=0.9$, and $\Lambda=0.6$ (solid line). Zimm-type [1] scaling $D \sim L^{-1 / 2}$ and $\tau_{r} \sim L^{3 / 2}$ (long dashed line). Rouse-type [1] scaling $D \sim L^{-1}$ and $\tau_{r} \sim L^{2}$ (short dashed line). Notes: For the two shortest DNA fragments, the intramolecular relaxation times could not be reliably resolved and are not presented. To account for the difference in experimental techniques, the TEB relaxation times from [8] are multiplied by a factor of 2. Insets show the ratios of our experimental results to the prediction of the semiflexible polymer theory. 
Recently, several FCS-based studies of dsDNA dynamics in solution have been published [10-12]. In experiments $[10,12]$ the interpretation of FCS data in terms of segmental dynamics is more complicated due to multiple labeling of DNA molecules; additionally, in [10] a powerlaw approximation for intramolecular DNA dynamics was used over the whole time range. In study [11], Rouse-type dynamics was observed in the mean-square displacement of end-labeled dsDNA fragments, which was attributed to weakness of hydrodynamic interactions for dsDNA in dilute solution. This explanation contradicts previous knowledge and theoretical predictions, as well as our present results. Unfortunately, no experimental dependences of $D$ and $\tau_{r}$ on the dsDNA length were obtained in [11] to support this unexpected finding. We conjecture that the method of producing dsDNA samples in [11] could yield a considerable amount of unlabeled DNA molecules, thus resulting in a semidilute solution characterized by Rouse-type dynamics [1].

In conclusion, diffusion and segmental dynamics of dsDNA have been quantitatively studied over the transition range from stiff to semiflexible chains by applying fluorescence correlation spectroscopy to single-end fluorescently labeled monodisperse DNA fragments. FCS correlation functions, diffusion coefficients, and longest macromolecular relaxation times of dsDNA fragments with lengths in the range of 0.1-20 kbp are in excellent agreement with the theory of semiflexible polymers [4] and clearly demonstrate that hydrodynamic interactions control the segmental dynamics of dsDNA.

Financial support by the Deutsche Forschungsgemeinschaft within the Priority Program SPP 1164 is gratefully acknowledged. We are grateful to Dr. B. Tinland for making accessible data of Ref. [7] and to K. Birkenfeld for her invaluable assistance with DNA samples.

[1] M. Doi and S.F. Edwards, The Theory of Polymer Dynamics (Clarendon Press, Oxford, 1986).

[2] R. Pecora, Science 251, 893 (1991).

[3] V. A. Bloomfield, D. M. Crothers, and I. Tinoco, Jr., Nucleic Acids: Structures, Properties, and Functions (University Science Books, Sausalito, CA, 2000).

[4] L. Harnau, R. G. Winkler, and P. Reineker, J. Chem. Phys. 104, 6355 (1996).

[5] (a) W. Eimer and R. Pecora, J. Chem. Phys. 94, 2324 (1991); (b) S. S. Sorlie and R. Pecora, Macromolecules 23, 487 (1990).

[6] D. E. Smith, T. T. Perkins, and S. Chu, Macromolecules 29, 1372 (1996).

[7] A.E. Nkodo, J. M. Garnier, B. Tinland, H. Ren, C. Desruisseaux, L.C. McCormick, G. Drouin, and G. W. Slater, Electrophoresis 22, 2424 (2001).

[8] (a) R. J. Lewis, R. Pecora, and D. Eden, Macromolecules 19, 134 (1986); (b) Y. Lu, B. Weers, and N. C. Stellwagen, Biopolymers 61, 261 (2002).

[9] P. Schwille, Cell Biochem. Biophys. 34, 383 (2001).
[10] D. Lumma, S. Keller, T. Vilgis, and J. O. Rädler, Phys. Rev. Lett. 90, 218301 (2003).

[11] R. Shusterman, S. Alon, T. Gavrinyov, and O. Krichevsky, Phys. Rev. Lett. 92, 048303 (2004).

[12] R. G. Winkler, S. Keller, and J. O. Rädler, Phys. Rev. E 73, 041919 (2006).

[13] R. K. Saiki, D. H. Gelfand, S. Stoffel, S. J. Scharf, R. Higuchi, G. T. Horn, K. B. Mullis, and H. A. Erlich, Science 239, 487 (1988).

[14] Primers are oligonucleotides defining the sequence and length of the DNA fragment to be amplified in the PCR.

[15] N. Panchuk-Voloshina, R. P. Haugland, J. Bishop-Stewart, M. K. Bhalgat, P. J. Millard, F. Mao, W.-Y. Leung, and R. P. Haugland, J. Histochem. Cytochem. 47, 1179 (1999).

[16] HotMaster Taq DNA Polymerase (0.1-5 kbp fragments) and TripleMaster PCR System (Eppendorf) (10 and $20 \mathrm{kbp}$ fragments) with the producer-specified error rates of $1.25 \times 10^{-5}$ and $2.30 \times 10^{-6}$, respectively, were used to perform PCR in a Mastercycler ep gradient $S$ thermocycler (Eppendorf). DNA fragments with the lengths of 0.1 to $5 \mathrm{kbp}$ were twice gel purified with $0.8 \%(w / v)$ agarose gels and isolated with the NucleoSpin Extract II kit (Macherey-Nagel $\mathrm{GmbH} \& \mathrm{Co}$. KG). To avoid shearing-induced damage, the 10 and $20 \mathrm{kbp}$ DNA fragments were gel purified and electroeluted with D-Tube Dialyzer Midi (MWCO $3.5 \mathrm{kDa}$ ) and D-Tube Electroelution Accessory Kit (Novagen). Samples were immediately frozen and stored at $-20{ }^{\circ} \mathrm{C}$. Measurements were carried out in $5 \mathrm{~m} M$ Tris- $\mathrm{HCl}(p \mathrm{H} \mathrm{8.0)}, 1 \mathrm{~m} M$ EDTA, $0.1 M \mathrm{NaCl}$ in LiChrosolv water (Merck).

[17] R. Rigler, Ü. Mets, J. Widengren, and P. Kask, Eur. Biophys. J. 22, 169 (1993).

[18] K. Weisshart, V. Jungel, and S. J. Briddon, Curr. Pharm. Biotechnol. 5, 135 (2004).

[19] J. Widengren, Ü. Mets, and R. Rigler, J. Phys. Chem. 99, 13368 (1995)

[20] C. Eggeling, A. Volkmer, and C. A. M. Seidel, Chem. Phys. Chem. 6, 791 (2005).

[21] M. Zhao, L. Jin, B. Chen, Y. Ding, H. Ma, and D. Chen, Appl. Opt. 42, 4031 (2003).

[22] Concentrations of $0.1,0.2,0.5,1,2,5,10$, and $20 \mathrm{kbp}$ DNA fragments were estimated as 11.6, 11.3, 19.3, 21.2, $3.5,2.2,1.7$, and $1.7 \mathrm{nM}$, respectively.

[23] C.T. Culbertson, S.C. Jacobson, and J. M. Ramsey, Talanta 56, 365 (2002).

[24] Function ROBUSTFIT from the Statistics Toolbox 5.0 of MATLAB 7.0 (The Matworks, Inc.) was used to estimate $G(0)$ with an accuracy of about $1 \%$ by fitting a constant to $G(t)$ values in the time interval of $0.5-1.5 \mu \mathrm{s}$.

[25] R. G. Winkler, J. Chem. Phys. 118, 2919 (2003).

[26] G. Allegra and F. Ganazzoli, J. Chem. Phys. 74, 1310 (1981).

[27] Using $\langle N\rangle$ as an additional fitting parameter leads to just a few percent changes in the least-square estimates of $l_{p}, \Lambda$, and $\Lambda_{D}$, and therefore of $D$ and $\tilde{\tau}_{l}$.

[28] The values of diffusion coefficients are essentially model independent, since they are determined by varying $\Lambda_{D}$ from the tails of the correlation functions, where the intramolecular contribution to the MSD is saturated.

[29] In the absence of hydrodynamic interactions, the Rousetype scaling $D \sim L^{-1}$ is expected [1]. 